\title{
Enhanced beam characteristics of a discharge-pumped soft-x-ray amplifier by an axial magnetic field
}

\author{
F. G. Tomasel, ${ }^{*}$ V. N. Shlyaptsev, ${ }^{\dagger}$ and J. J. Rocca \\ Department of Electrical Engineering, Colorado State University, Fort Collins, Colorado 80523
}

(Received 26 January 1996)

\begin{abstract}
We report results of the study of soft-x-ray amplification in a discharge-created plasma under an externally applied axial magnetic field. This technique is observed to improve the uniformity and divergence of the beam of a 46.9-nm discharge-pumped Ne-like Ar soft-x-ray amplifier by decreasing the plasma density gradients at the time of lasing. At the optimum strength of $0.15 \mathrm{~T}$, the magnetic field also causes an increase in the laser beam intensity. At higher fields the Zeeman effect is found to contribute to line broadening, decreasing the laser output. [S1050-2947(96)11609-7]
\end{abstract}

PACS number(s): 42.55.Vc

\section{INTRODUCTION}

There is significant interest in the development of compact and efficient soft-X-ray laser sources [1,2]. A promising approach consists in the use of fast discharges to excite the gain medium. The first demonstration of large soft-x-ray amplification in a discharge-driven plasma was recently realized using a fast capillary discharge to generate a hot and dense plasma column in which collisional electron excitation of Ne-like Ar ions produced amplification in the $J=0-1$ line of Ne-like Ar at $46.9 \mathrm{~nm}$ [3-5]. In this excitation scheme, a fast current pulse rapidly compresses the plasma, creating a hot and narrow plasma column with length-to-diameter ratios approaching 1000:1. During the final stage of the compression, plasma conditions for soft-x-ray amplification by collisional excitation are obtained. In the initial experiments, a gain-length product of $g l \sim 7.2$ at $46.9 \mathrm{~nm}$ was reported for a 12-cm-long plasma column. Subsequent experiments conducted at optimized plasma conditions demonstrated exponential amplification for plasma column lengths up to $15 \mathrm{~cm}$, yielding gain-length products up to $g_{l} \sim 14$, value approaching that necessary to saturate the gain of the amplifier [6].

In those experiments, the 46.9-nm laser beam pattern was observed to have divergences that are larger than the value determined by the geometry of the plasma column at the time of lasing. In some cases the laser pattern was observed to present two off-axis side lobes, corresponding to an annular beam profile [6]. This is indicative of refraction of the amplified radiation as a result of steep plasma density gradients at the time of lasing. Refraction decreases the effective gain coefficient and, in an extreme case, can terminate the exponential amplification, limiting the maximum gain-length product that could be obtained [7-10]. Our results show that these detrimental effects can be reduced by embedding the plasma column into an axial magnetic field, which decreases the plasma density gradients while simultaneously maintaining the adequate plasma conditions necessary for large amplification.

\footnotetext{
*On leave from Facultad de Ingenieria, Universidad Nacional de Mar del Plata, Argentina.

${ }^{\dagger}$ On leave from P. N. Lebedev Phys. Inst., Moscow, Russia.
}

Axial magnetic fields can also be expected to contribute to further enhance the axial uniformity of the amplifying plasma column. Externally applied axial magnetic fields have been successfully used in the stabilization of the dynamics of different axially imploded discharges [11-14]. In the particular case of capillary discharges, the effects of strong magnetic fields $(\sim 7 \mathrm{~T})$ in the recombination phase of an ablative capillary discharge have been previously studied [15]. In X-ray laser research, it has been suggested that axial magnetic fields could be used to stabilize inhomogeneities that have precluded the use of some $z$-pinch discharges as successful amplifiers $[12,13,16]$. Magnetic fields have also been employed in laser-pumped soft-X-ray experiments. In this case, an intense axial magnetic field has been used to confine the plasma and maintain a high plasma density during the cooling phase of a $\mathrm{C}$ VI plasma recombination laser [17]. The demonstration of lasing at $18.2 \mathrm{~nm}$ in such magnetically confined laser-created plasma [17] motivated the study of the effect of external magnetic fields of up to $18 \mathrm{~T}$ on the x-ray emission characteristics of laser-produced plasmas [18].

In this paper we discuss the results of the study of soft-xray amplification in a discharge-created plasma column under the influence of an axial magnetic field. The magnetic field was observed to improve the uniformity and divergence of the soft-x-ray laser beam and to moderately increase the laser intensity. Hydrodynamic calculations suggest that this enhancement in the laser beam characteristics is due to reduction in the density gradients in the gain region of the amplifier.

\section{EXPERIMENTAL SETUP}

The experiments were conducted in a capillary discharge excited, collisionally pumped 46.9-nm Ne-like Ar amplifier. To conduct the study, we modified a fast capillary discharge setup previously described in Ref. [4] to include an axial magnetic field. The discharge consists of a 3-nF capacitor which is pulse-charged by a Marx generator and is discharged through a pressurized $\mathrm{SF}_{6}$ switch into a capillary channel containing a selected pressure of preionized argon gas. In the experiments described herein, the generator was used to excite plasmas in polyacetal capillaries $4 \mathrm{~mm}$ in di- 
ameter and $10 \mathrm{~cm}$ in length with current pulses having a first half cycle duration of approximately 64 ns. The axial magnetic field was generated by a $9-\mathrm{cm}$ diameter, $15-\mathrm{cm}$ long coil positioned concentrically with the capillary channel. The coil, which was excited by a current pulse with a period of $200 \mu$ s obtained by discharging a $420-\mu \mathrm{F}$ capacitor through a spark gap, was used to produce magnetic fields up to $0.3 \mathrm{~T}$. The intensity of the magnetic field was selected by varying either the capacitor charging voltage or the delay time between the triggering of the latter spark gap and the firing of the fast capillary discharge. The discharge electrodes were made of stainless steel and were slotted to improve the uniformity of the magnetic field along the capillary axis, which was measured, using an axial Hall effect probe, [19] to be better than $20 \%$ over the $10-\mathrm{cm}$-long region where the capillary is located. The dc calibration of Hall probe given by the manufacturer, $0.1146 \mathrm{~V} / \mathrm{T}$, was checked in the pulsed mode by placing it on the axis and in the center of the coil described above and comparing the measured value with the one calculated from the expression for a finite solenoid.

The soft-x-ray radiation exited the capillary through the hollowed ground electrode. The laser radiation was collected by a cylindrical copper mirror of $13 \mathrm{~cm}$ in radius and focused onto the slit of a $2.2-\mathrm{m}$ vacuum spectrometer provided with a $1200 \mathrm{l} / \mathrm{mm}$ diffraction grating placed at $4.2^{\circ}$ with respect to the incoming radiation. The detection system consisted of an intensified charge-coupled device (CCD) array detector that was gated by pulsing the gain on the multichannel plate intensifier with a high voltage pulse with a duration of about $25 \mathrm{~ns}$.

\section{RESULTS AND DISCUSSION}

The effects of the axial magnetic field on the intensity profile of the laser beam is illustrated in Fig. 1. Figures 1(a) and 1(c) show beam patterns recorded along the direction of the spectrometer slit for the condition of no external magnetic field applied. The beam profile presents either a single or double peaks, depending on the particular characteristics of the discharge shot which can result in different degrees of plasma compression and gain distribution profiles. In both cases, however, the beam divergence is larger the $\leqslant 3 \mathrm{mrad}$ which can be expected from the geometry of the amplifying plasma column at the time of lasing $(\leqslant 300 \mu \mathrm{m}$ diameter, 10 $\mathrm{cm}$ length). Such broadening of the beam profile can be attributed to refraction of the amplified soft-x-ray radiation due to plasma density gradients in the gain region. The consequences of refraction have been previously analyzed, and are well known [7-9]. Refraction bends the rays off the gain region, causing a decrease in the effective gain coefficient that depends on the density gradient [7]. In a plasma with cylindrical geometry, such as our capillary discharge, and for sufficiently long plasma columns, refraction reduces the gain coefficient by a factor $\left(1-2 / G_{r}\right)$, where $G_{r}$ depends on the gain $g\left(r_{m}\right)$ and on the curvature of the index of refraction at the position of maximum density, $r_{m}$, as $G_{r}=g\left(r_{m}\right) / \sqrt{\partial^{2} n / \partial r^{2}}$ [8]. The angular separation of the side lobes can be shown to be related to the electron density at the time of lasing $[7,8]$. Therefore, it provides with a measurement of the electron density in the gain region at the time of lasing. The separation measured in Fig. 1(a), approximately
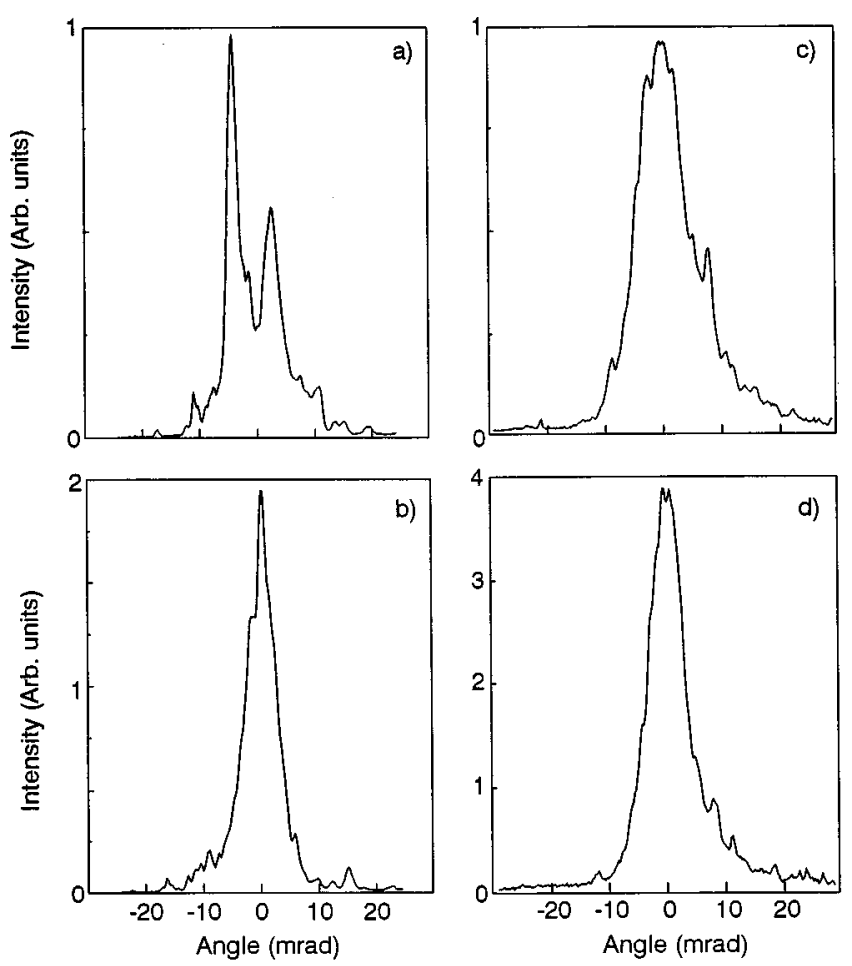

FIG. 1. Soft-X-ray laser beam profiles $(\lambda=46.9 \mathrm{~nm})$ corresponding to discharges of approximately $39 \mathrm{kA}$ through $10-\mathrm{cm}-$ long, 4-mm-diam capillary channels filled with 600 mTorr of argon. (a) and (c) With no external magnetic field applied. (b) and (d) With an axial magnetic field of $0.15 \mathrm{~T}$.

$5.7 \mathrm{mrad}$, corresponds to an electron density of about $4 \times 10^{18} \mathrm{~cm}^{-3}$, value which is in good agreement with that resulting from our hydrodynamic simulation of the argon capillary discharge plasma. Figures 1(b) and 1(d) correspond to shots conducted after shots 1 (a) and 1(c), respectively, with an externally applied magnetic field of $0.15 \mathrm{~T}$. In both cases the magnetic field is observed to decrease the beam divergence, in the first case also significantly improving the beam uniformity. These results are consistent with reduced refraction effects, associated with smoother electron density profiles.

Hydrodynamic computations of the evolution of the plasma column with and without an externally applied magnetic field were performed utilizing a hydrodynamic-atomic code specifically developed to model the fast capillary discharge [20]. The numerical code, which models all major aspects of $\mathrm{x}$-ray laser physics, it is based on that previously described in Ref. [20]. It is based on classical 1D hydrodynamic equations but modified to include the results of turbulent and anomalous plasma kinetic theory. The code is self-consistently coupled to a non-local-thermodynamicequilibrium atomic physics model that calculates the kinetics of the ionization and excitation of ions and radiation transport. To model the capillary discharge plasma, the influence of ablated wall material in the plasma dynamics was also taken into account. Electrodynamical and hydrodynamical processes, including magnetic diffusion and dissipative processes connected with the externally applied magnetic field, were self-consistently treated.

The simulations show that an externally applied axial magnetic field of $0.15 \mathrm{~T}$, due to its relatively small value, 


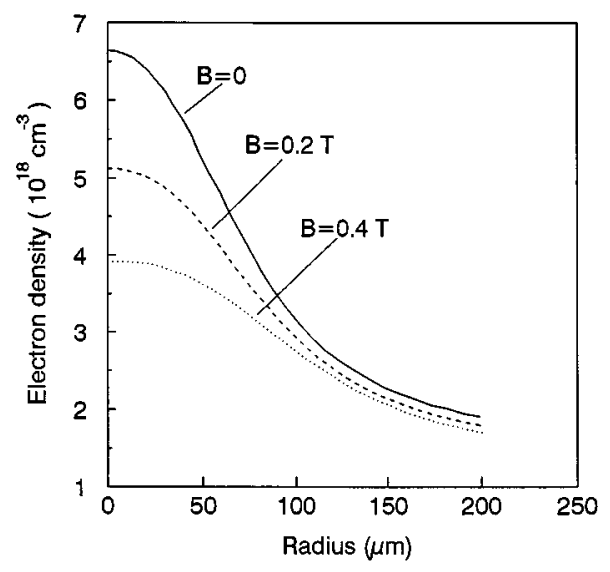

FIG. 2. Computed electron density profiles on the axial magnetic region of the plasma column at the time of lasing, for externally applied magnetic fields with strengths of $0,0.2$, and $0.4 \mathrm{~T}$. The results are for a capillary diameter of $4 \mathrm{~mm}$ excited by a $39-\mathrm{kA}$ current pulse and filled with 600 mTorr of argon.

does not significantly affect the evolution of the plasma column until the final stage of the implosion, when the magnetic field is strongly compressed reaching values of the order of $10 \mathrm{~T}$. For example, the model predicts that the time of maximum compression and that for lasing are not significantly changed by the magnetic field. This is in agreement with the experimental observation that the time of lasing in discharges with and without a 0.15-T axial magnetic field is the same within the error of the measurement, \pm 2 ns. Nevertheless, the simulations also show that at the time at which the amplification occurs the radial density profile is smoother in presence of the axial magnetic field. This behavior may be understood based on the simple consideration that, for the

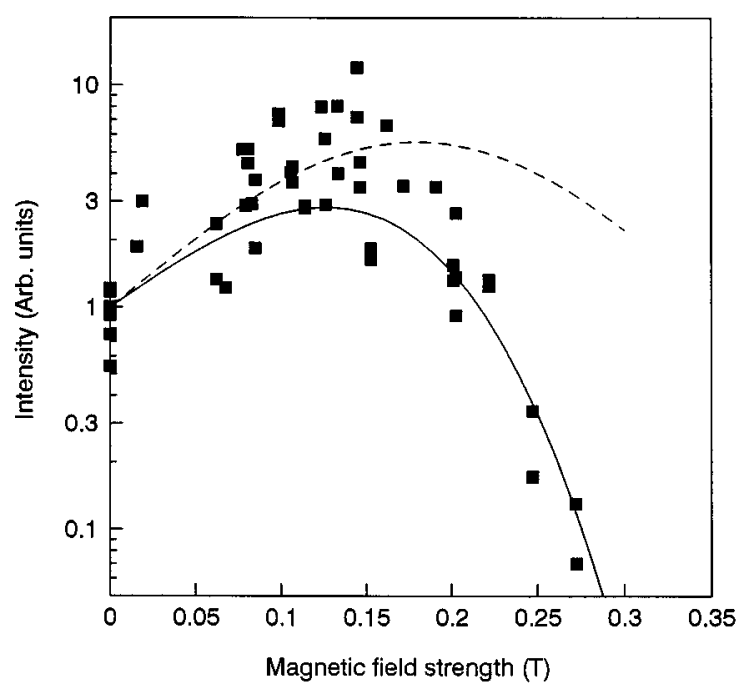

FIG. 3. Variation of the integrated intensity of the 46.9-nm Ar IX laser line as a function of the strength of the externally applied axial magnetic field. The solid markers correspond to data from discharges through 10-cm-long, 4-mm-diam capillaries filled with approximately $600 \mathrm{mTorr}$ of argon. The half cycle width of the current pulse is $64 \mathrm{~ns}$. The solid and dashed curves are the result of computations with and without including Zeeman splitting, respectively.
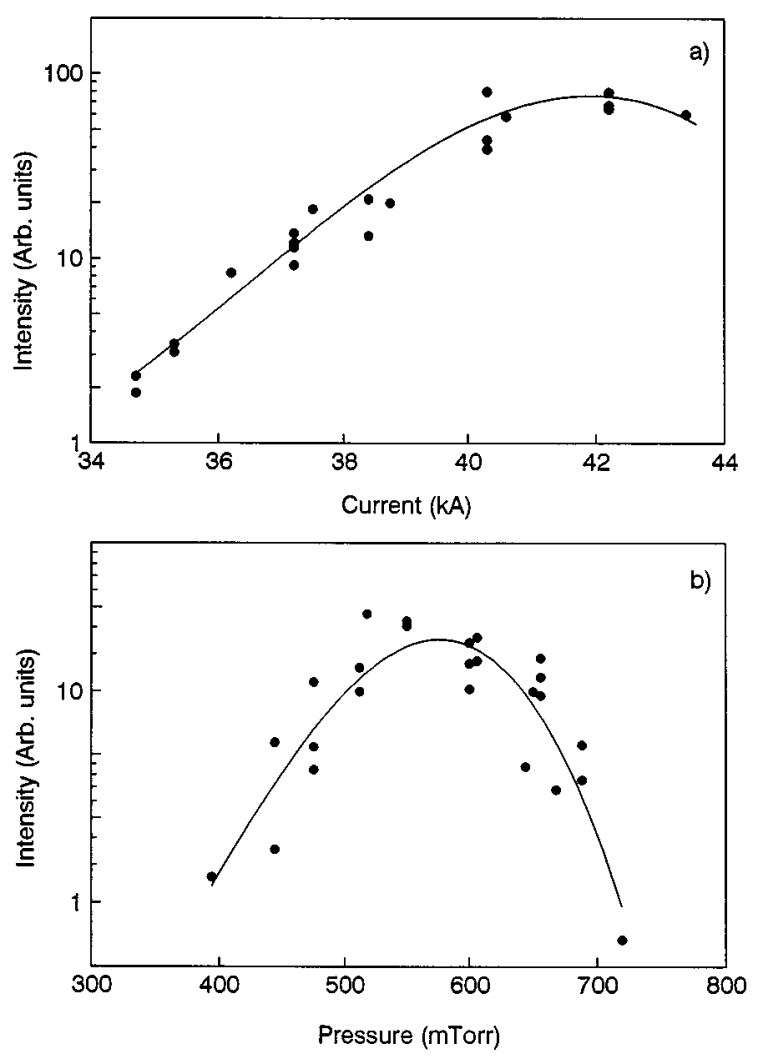

FIG. 4. Integrated intensity of the 46.9-nm Ar IX laser line as a function of peak current (a) and filling pressure (b), for discharges with an external magnetic field of $0.15 \mathrm{~T}$. The data in (a) correspond to the optimum pressure measured on (b) (approximately 600 mTorr), while the discharge current on (b) was approximately 39 kA.

discharge parameters used in our experiment, the compressed axial magnetic field, $\propto B_{z} / r^{2}$, reaches the same magnitude as the self-generated azimuthal magnetic field, $\propto I / r$, only near the end of the plasma compression. As a reference point, one can obtain a crude estimate of the minimum value of the externally applied magnetic field necessary to appreciably modify the electron density gradient at the time of lasing, close to the stagnation point, by equating the pressure of the compressed axial magnetic field, $B_{z}^{2} / 2 \mu_{0}$, to the plasma kinetic pressure, $\left(N_{e} T_{e}+N_{i} T_{i}\right)$. Such a simple estimation gives a compressed magnetic field value of about $10 \mathrm{~T}$ which, assuming a radial compression ratio of 15 and neglecting diffusion of the magnetic field, corresponds to externally applied fields of the order of $0.05 \mathrm{~T}$.

Figure 2 shows typical computed electron density profiles at the time of maximum laser power generation for three different values of the magnetic field: $0,0.2$, and $0.4 \mathrm{~T}$. The computed electron density gradient is observed to decrease as a function of the increasing magnetic field, which is in concordance with the observation of reduced refraction.

The variation of the measured integrated intensity of the Ar IX 46.9-nm laser line as a function of the magnetic field strength is shown in Fig. 3. The laser intensity increases with the magnetic field and reaches a maximum at approximately $0.15 \mathrm{~T}$ decreasing monotonically for higher field strengths. The same figure also shows the calculated variation of the intensity corresponding to two calculations which differ from 
each other in the inclusion of Zeeman splitting of the laser line. In our case, the Zeeman splitting is mostly caused by the compressed axial magnetic field. The azimuthal magnetic field is comparatively small in the gain region, situated near the axis, due to the distribution of the current and the boundary condition $B_{\theta}=0$ at $r=0$. In both computations the laser intensity first increases to subsequently decrease at higher values of the magnetic field. The intensity increase is due to decreased refraction losses and to a larger electron temperature caused by a decrease in heat losses. Only the computation that considers broadening of the laser line due to the Zeeman effect shows good agreement with the experiment. In the condition of our experiments the line profile is largely determined by Doppler broadening, and collisional and natural broadening contributions are small. Moderate magnetic fields, of the order of $10 \mathrm{~T}$, contribute to broaden the $2 p^{5} 3 s^{1} S_{0}-2 p^{5} 3 p^{1} P_{1}$ transition of Ne-like argon by $\Delta \nu / \nu \sim 5 \times 10^{-5}$. This value is comparable to the Doppler linewidth of the order of $\Delta \nu / \nu \sim 1 \times 10^{-4}$, therefore causing a very significant decrease in the measured $g_{l}$ product. These results suggest that the Zeeman effect is likely to be a major cause for the observed decrease of the laser output intensity at higher magnetic fields. The laser intensity decrease observed at large magnetic fields in the computation that excludes the Zeeman effect is the result of a smaller gain caused by a decrease in the density, reduced transient effects associated with ionization and excitation, and an increase in the optical depth. The larger optical depth at higher magnetic fields is due to a reduction of the very important radial motional Doppler effect, which is in turn caused by the previously discussed reduction of the density gradients.

At the optimum value, the axial magnetic field is observed to increase the 46.9-nm laser line intensity produced by a 10 -cm-long plasma column by a factor of 3-4 respect to that obtained without an external magnetic field. This intensity enhancement corresponds to an increase in the gain-length product of about unity, meaning that for plasma column length used in these experiments the increase in the effective gain amounts to about $0.1 \mathrm{~cm}^{-1}$. Since in experiments con- ducted in 4-mm-diam capillaries at similar discharge conditions we have measured gain coefficients of approximately 1 $\mathrm{cm}^{-1}$ [6], the observed intensity increase corresponds to an increment of about $10 \%$ in the gain coefficient of the 46.9-nm Ar IX line.

Figures 4(a) and 4(b) show the dependence of the integrated laser intensity on the peak discharge current and on the filling pressure, at the optimum value of the external magnetic field strength shown in Fig. 3. The variation of the intensity as a function of these two parameters is similar to the one observed for discharges with no external field applied [6]. The intensity shows a maximum for currents close to $42 \mathrm{kA}$, current that is slightly higher than the optimum value for the case of zero field [5], and decreases monotonically for both lower and higher currents. Figure 4(b) shows that optimum conditions for lasing in the presence of the externally applied magnetic field are achieved when the pressure is close to 600 mTorr.

\section{SUMMARY}

In summary, we have demonstrated a technique to govern the characteristics of discharge-pumped soft-x-ray lasers. An externally applied axial magnetic field of approximately 0.15 $\mathrm{T}$ is shown to affect the hydrodynamics and kinetics of the compressional plasma at the time of lasing, improving the beam profile characteristics and increasing the output intensity of a Ne-like argon soft-x-ray laser. The results also suggest that the Zeeman effect contributes to decreasing the laser output at higher magnetic fields.

\section{ACKNOWLEDGEMENTS}

We thank A. Osterheld for providing the atomic data utilized in the computations, and L. A. Vainstein and J. L. A. Chilla for helpful discussions. This work was supported by NSF Grants Nos. ECS 9401952 and ECS 9412106. Part of the diagnostics instrumentation was developed in collaboration with Hyperfine Inc. (Boulder, CO) with the support of the Colorado Advanced Technology Institute.
[1] Proceedings of the Fourth International Colloquium on X-Ray Lasers, edited by D. Eder and D. Matthews, AIP Conf. Proc. No. 332 (AIP, New York, 1994).

[2] J. J. Rocca and P. L. Hagelstein, in Soft X-ray Lasers and Applications, edited by J. J. Rocca and P. L. Hagelstein, special isue of SPIE J. 2520 (1995).

[3] J. J. Rocca, V. N. Shlyaptsev, F. G. Tomasel, O. D. Cortázar, D. Hartshorn, and J. L. A. Chilla, Phys. Rev. Lett. 73, 2192 (1994).

[4] J. J. Rocca, O. D. Cortázar, B. Szapiro, K. Floyd, and F. G. Tomasel, Phys. Rev. E 47, 1299 (1993).

[5] J. J. Rocca, F. G. Tomasel, M. C. Marconi, V. N. Shlyaptsev, J. L. A. Chilla, B. T. Szapiro, and G. Giudice, Phys. Plasmas 2, 2547 (1995).

[6] J. J. Rocca, M. C. Marconi, J. L. A. Chilla, D. P. Clark, F. G. Tomasel, and V. N. Shlyaptsev, IEEE J. Select. Topics Quantum Electron. 1, 945 (1995).
[7] R. A. London, Phys. Fluids 31, 184 (1988).

[8] V. A. Chirkov, Sov. J. Quantum Electron. 14, 1497 (1984).

[9] J. L. A. Chilla and J. J. Rocca (unpublished).

[10] V. N. Shlyaptsev and P. N. Levedev, Physics Institute Report No. 268, 1984 (unpublished); P. N. Levedev, Ph.D. thesis, Physics Institute, 1987 (unpublished).

[11] S. Glasstone and R. H. Lovberg, Controlled Thermonuclear Reactions (Van Nostrand, Princeton, NJ, 1960).

[12] F. S. Felberg, F. J. Wessel, N. C. Weld, H. U. Rahman, A. Fisher, C. M. Fowler, M. A. Liberman, and A. L. Velikovich, J. Appl. Phys. 64, 3831 (1988).

[13] S. A. Sorokin, A. V. Khachaturyan, and S. A. Chaikovskii, Fiz. Plasmii 17, 1453 (1991) [Sov. J. Plasma Phys. 17, 841 (1992)].

[14] N. S. Edison, B. Etlecher, A. S. Chuvatin, S. Attelan, and R. Aliaga, Phys. Rev. E 48, 3893 (1993).

[15] M. C. Marconi, J. J. Rocca, J. F. Schmerge, M. Villagran, and F. Lehmann, IEEE J. Quantum Electron. 26, 1809 (1990). 
[16] V. N. Shlyaptsev and A. V. Gerusov, in Proceedings of the Third International Colloquium on X-ray Lasers, edited by E. E. Fill, IOP Conf. Proc. No. 125 (Institute of Physics and Physical Society, London, 1992), p. 125.

[17] S. Suckewer, C. H. Skinner, H. Milchberg, C. Keane, and D. Voorhees, Phys. Rev. Lett. 55, 1753 (1985).

[18] H. Fiedorowicz, B. A. Brynetkin, A. Ya. Faenov, A. Farynsky,
R. Miklaszewski, M. Mroczkowski, P. Parys, T. Pisarczyk, I. Yu. Skobelev, and M. Szczurek, in Proceedings of the Third International Colloquium on X-ray Lasers (Ref. [16]), p. 415.

[19] F. W. Bell, Axial Hall generator model BH-203.

[20] V. N. Shlyaptsev, A. V. Gerusov, A. V. Vinogradov, J. J. Rocca, O. D. Cortazar, F. Tomasel, and B. Szapiro, SPIE J. 2012, 99 (1993). 